\title{
ОБЕСПЕЧЕНИЕ БАЛАНСОВОЙ НАДЕЖНОСТИ ЭЛЕКТРОЭНЕРГЕТИЧЕСКИХ СИСТЕМ С ВОЗОБНОВЛЯЕМЫМИ ИСТОЧНИКАМИ ЭНЕРГИИ
}

\author{
С.В. Казанский, канд. техн. наук, доцент, Ю.П. Матеенко, канд. техн. наук, доцент
}

Национальный технический университет Украины «Киевский политехнический институт им. Игоря Сикорского» 03056 г. Киев, пр-т Победы, 37

Дано определение оперативной и балансовой надежности как двух составляющих надежности функционирования электроэнергетических систем (ЭЭС). Отмечено, что балансовая надежность - это способность ЭЭС постоянно поддерживать баланс активной мощности и обеспечивать выдачу мощности, необходимой потребителям, с учетом вероятных отключений элементов ЭЭС. Показана необходимость обеспечения балансовой надежности ЭЭС и энергообъединений как единого объекта управления. При этом балансовая надежность является объективной характеристикой состояния генерирующей части ЭЭС и критерием вероятности ограничения выдачи мощности потребителям. Показана взаимосвязь увеличения количества возобновляемых источников энергии и возрастания вероятности снижения балансовой надежности ЭЭС с ограничениями по мощности генерирующих агрегатов или по пропускной способности электрических сетей. Приведены показатели определения балансовой надежности ЭЭС. Показана последовательность определения вероятности непокрытия нагрузки системы, основанная на использовании марковской модели генерирования мощности. Предложена методика численной оченки показателей балансовой надежности ЭЭС с возобновляемыми источниками энергии. Рассмотрены модели представления нагрузки характеристиками случайной величины или случайного процесса. Выполнен сравнительный анализ моделей при определении балансовой надежности. Показано, что в условиях функиионирования рынка электрической энергии возрастает вероятность нарушения балансовой надежности ЭЭС. Предложена упрощенная методика оценки балансовой надежности для ЭЭС со значительным количеством возобновляемых источников энергии, которая позволяет по значениям вероятности отказа системь оценить вероятность непокрытия нагрузки на произвольном интервале времени, а также дефицит мощности в ЭЭС. Приведена графическая интерпретация упрощенной модели обеспечения балансовой надежности ЭЭС. Библ. 4, рис. 5.

Ключевые слова: электроэнергетическая система, балансовая надежность, возобновляемые источники энергии, рынок электрической энергии.

\section{ENSURING BALANCE RELIABILITY OF ELECTRIC POWER SYSTEMS WITH RENEWABLE ENERGY SOURCES}

S. Kazanskiy, candidate of technical science, assistant professor, Y. Mateyenko, candidate of technical science, assistant professor

National Technical University of Ukraine «Igor Sikorsky Kyiv Polytechnic Institute»

03056, 37 Peremohy Avenue, Kyiv, Ukraine

The definition of operational and balance reliability as two components of the reliability of the operation of electrical power systems (EPS) is given. It was noted that book-based reliability is the ability of an EPS to constantly maintain the balance of active power and ensure the output of the power required by consumers, taking into account the likely outages of the EPS elements. The necessity of ensuring the balance reliability of EPS and energy interconnections as a single control object is shown. At the same time, the balance reliability is an objective characteristic of the state of the generating part of the EPS and the criterion of the probability of limiting the output of power to consumers. The relationship between increasing the number of renewable energy sources and increasing the likelihood of reducing the balance sheet reliability of an EPS with a limitation on the power of generating units or on the capacity of electrical networks is shown. The indicators for determining the reliability of the EPS are given. The sequence of determining the probability of not covering the system load, based on the use of the Markov power generation model, is shown. The technique of a numerical evaluation of indicators of the balance reliability of EPS with renewable energy sources is proposed. We consider the model representation of the load characteristics of a random variable or a random process. A comparative analysis of models in determining the balance of reliability. It is shown that under the conditions of functioning of the electric energy market the probability of breaches of the balance reliability of the EPS increases. A simplified method for estimating the balance reliability for an EPS with a significant amount of renewable energy sources is proposed, which allows estimating the probability of not covering the load over an arbitrary time interval, as well as the power deficit in an EPS. A graphical interpretation of a simplified model of the balance reliability of the EPS is given. Ref. 4, fig. 5 .

Keywords: electric power system, balance reliability, renewable energy sources, electric energy market.

(C) С.В. Казанский, Ю.П. Матеенко, 2019

Відновлювана енергетика. 2019. № 2 


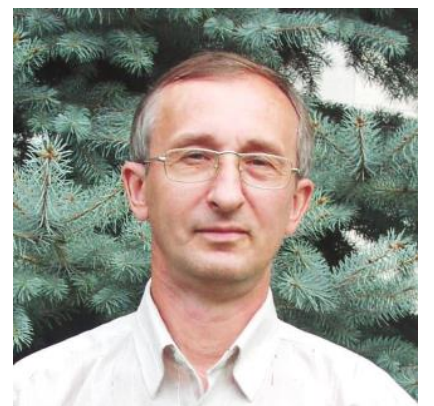

С.В. Казанский S. Kazanskiy

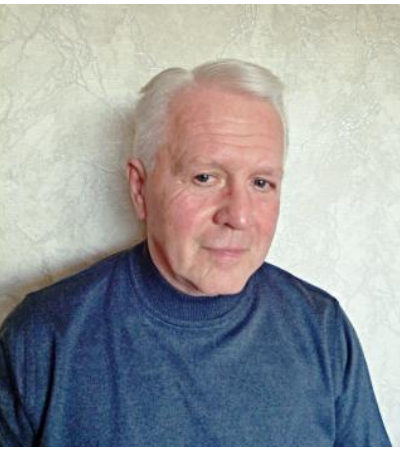

Ю.П. Матеенко Y. Mateyenko
Сведения об авторе: доцент кафедры электрических сетей и систем Национального технического университета Украины «Киевский политехнический институт имени Игоря Сикорского» (КПИ им. Игоря Сикорского), кандидат технических наук. Образование: Киевский политехнический институт, инженер-электрик по специальности «Электрические системы».

Научная сфера: Электрические сети и системы. Надежность электрических сетей и электроэнергетических систем. Повышение надежности и эффективности эксплуатации высоковольтного электрооборудования. Рынки электрической энергии. Реформирование рынка электрической энергии Украины.

Публикации: 140.

ORCID: 0000-0002-6113-2600

Контакти: +38 (044) 204-95-07

e-mail: elektropanorama@ukr.net

Сведения об авторе: доцент кафедры электрических станций Национального технического университета Украины «Киевский политехнический институт имени Игоря Сикорского» (КПИ им. Игоря Сикорского), кандидат технических наук.

Образование: Киевский политехнический институт, инженер-электрик по специальности «Электрические станции».

Научная сфера: Исследование проблем повышения надежности объектов электроэнергетики и обеспечение енергетической безопасности в условиях работы енергорынка.

Публикации: 72

ORCID: 0000-0002-2699-0888

Контакти: +38 (044) 206-28-09

e-mail: myurix@ukr.net
Information about the author: Associate Professor of the Department of Renewable Energy of the National Technical University of Ukraine «Igor Sikorsky Kyiv Polytechnic Institute» (Igor Sikorsky KPI), PhD of Technical sciences.

Education: Kiev Polytechnic Institute, engineer-electrical on the specialty «Electrical systems».

Research area: Electrical networks and systems. Reliability of electrical networks and power systems.

Improving the reliability and efficiency of operation of high-voltage electrical equipment. Electricity Markets.

Reforming the electricity market of Ukraine.

Publications: 140 .

ORCID: 0000-0002-6113-2600

Contacts: +38 (044) 204-95-07

e-mail: elektropanorama@ukr.net

Information about the author: Associate Professor of the Department of Power Stations of the National Technical University of Ukraine «Igor Sikorsky Kyiv Polytechnic Institute» (Igor sikorsky KPI), PhD of Technical sciences.

Education: Kyiv Polytechnic Institute, engineer-electrical on the specialty «Power Stations»y.

Research area: Investigation of the problems of improving the reliability of electric facilities and ensuring energy security in the operating conditions of the energy market.

Publications: 72 .

ORCID: 0000-0002-4798-6853

Contacts: +38 (044) 206-28-09

e-mail: myurix@ukr.net
Введение. Надежность электроэнергетических систем как единого объекта функционирования определяют их основные структурные подсистемы - генерирующая часть и системообразующая сеть, а также подсистемы управления режимами и ресурсного обеспечения [1].

Понятие надежности функционирования ЭЭС можно разделить на две составляющие оперативную и балансовую.

Оперативная надежность характеризует способность ЭЭС противостоять внезапным возмущениям и аварийным ситуациям.

Балансовая надежность - способность ЭЭС постоянно поддерживать баланс активной мощности и обеспечивать выдачу мощности, необходимой потребителям, с учетом вероятных отключений элементов ЭЭС [2].

В последние годы в Украине значительно возросло количество возобновляемых источников энергии (в основном солнечных и ветровых электрических станций). Увеличивается и суммарная установленная мощность указанных источников распределенной генерации, что приводит к необходимости дополнительной оценки балансовой надежности ЭЭС, поскольку возрастает вероятность перехода ЭЭС из состояния концентрированной в неконцентрированную [2].

Цель статьи - исследовать особенности определения балансовой надежности электроэнергетических систем, содержащих значительное количество возобновляемых источников энергии.

Материалы исследования Балансовую надежность ЭЭС оценивают такими показателями [2]:

- вероятность $p_{н}$ непокрытия нагрузки системы или ожидаемое за год количество суток $n_{t}$ непокрытой нагрузки;

- вероятность $p_{c}$ состояния отказа системы и среднее значение параметра потока отказов $Z_{c}$;

- усредненный за расчетный период $T$ дефицит мощности $P_{\partial}$ и недоотпущенная потребителям электроэнергия $W_{н}$. 
Построение модели генерирования мощности. Рабочая мощность системы в ее $m$-м состоянии, когда $m$ одинаковых агрегатов из общего количества $n$ отказали, составляет

$$
P_{\mathrm{c} m}=(n-m) P_{\text {ном }},
$$

где $P_{\text {ном }}-$ номинальная мощность одного агрегата.

Вероятность $p_{m} m$-го состояния определяется по формуле биноминального распределения

$$
p_{m}=C_{n}^{m} q^{m} p^{n-m},
$$

где $p, q-$ вероятности рабочего и нерабочего состояний агрегата соответственно.

Интенсивности переходов из $m$-го состояния в состояние $m-1$ большей рабочей мощности $\lambda_{m+}$ и в состояние $m+l$ меньшей рабочей мощности $\lambda_{m-}$ равны

$$
\lambda_{m+}=m \lambda_{\mathrm{B}} ; \lambda_{m-}=(n-m) \lambda,
$$

где $\lambda, \lambda_{\beta},-$ интенсивности соответственно отказов и восстановления агрегата.

Параметр потока (частоту) возникновения $m$ го состояния определяют как

$$
\begin{aligned}
& Z_{m}=p_{m}\left(\lambda_{m+}+\lambda_{m-}\right)= \\
& =p_{m}\left(m \lambda_{\mathrm{B}}-m \lambda+n \lambda\right) .
\end{aligned}
$$

Рабочая мощность системы в элементарном состоянии $P_{\ni}$ равна сумме номинальных

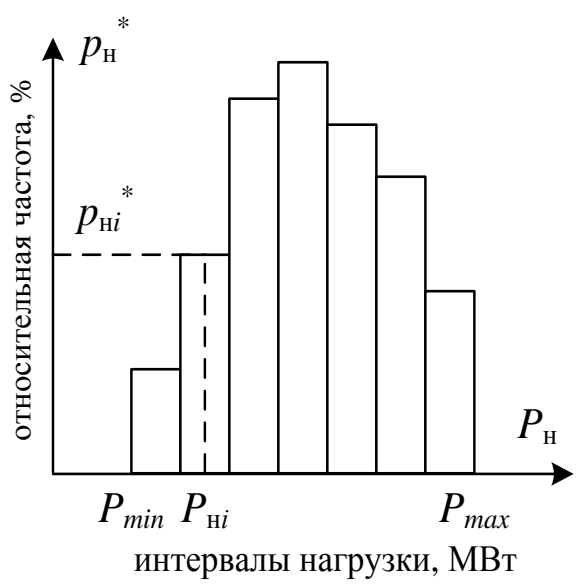

$a$ мощностей агрегатов, которые в этом состоянии системы работают. Вероятности элементарных состояний определяют по формуле

$$
p_{\ni}=\prod_{i=1}^{m} q_{i} \prod_{i=m+1}^{n} p_{i} .
$$

Интенсивности переходов между объединенным и элементарными состояниями и между двумя объединенными определяют по формулам:

$$
\lambda_{i J}=\sum_{j \in J} \lambda_{i j} ; \quad \lambda_{J i}=\sum_{j \in J} p_{j} \lambda_{j i} / \sum_{j \in J} p_{j}
$$

где $p_{i}, p_{j}-$ вероятности возникновения $i$-го и $j$-го элементарных состояний; $p_{J}-$ вероятность возникновения $J$-го объединенного состояния.

Параметр потока (частоту) возникновения $s$-го состояния определяют как

$$
Z_{s}=p_{s} \sum_{j \neq s} \lambda_{s j}
$$

Построение модели нагрузки. При анализе балансовой надежности ЭЭС нагрузки задают характеристиками случайной величины или случайного процесса. В первом случае ее представляют в виде гистограммы относительных частот нагрузки (рис. 1, а) или в виде графика нагрузки по продолжительности (рис. 1, б).

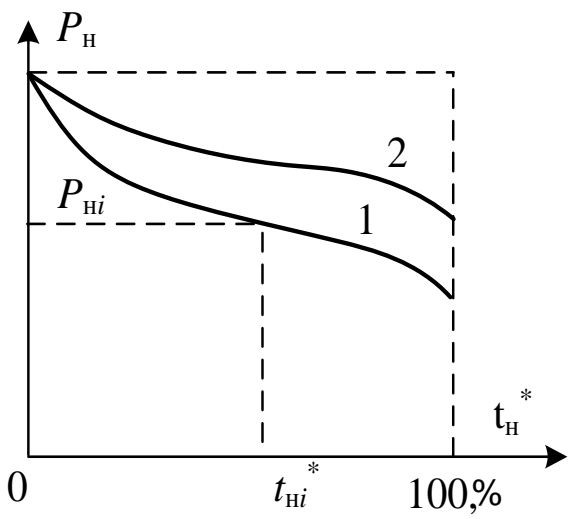

относительное время, \%

$$
\sigma
$$

Рис. 1. Гистограмма относительных частот нагрузки (a) и график нагрузки по продолжительности (б)

Fig. 1. The histogram of the relative frequency of the load $(a)$ and the load graph for the duration $(b)$

Относительная частота $p_{\text {н }}^{*}-$ это процент нагрузки, значение которого попало в заданный интервал. Она позволяет определить вероятность среднего для $i$-го интервала дискретного значения нагрузки $P_{\text {нi }}$ как

$$
p\left(P_{\mathrm{н} i}\right)=p_{\mathrm{н} i}^{*} / 100 .
$$


C помощью графика нагрузки по продолжительности можно определить вероятность превышения заданного значения мощности нагрузки

$$
p\left(P_{\mathrm{H}}>P_{\mathrm{H} i}\right)=t_{\mathrm{H} i}^{*} / 100 .
$$

Представление нагрузки характеристиками случайного процесса представляется намного сложнее. На рис. 2, $а$ показан типовой суточный двухуровневый график нагрузки, а на рис. 2, б показана его простейшая двухуровневая модель (когда нагрузку задают постоянным минимальным значением $P_{\text {н0 }}$ и случайно изменяющимся суточным максимальным значением $P_{\text {нi }}$. Изменения нагрузки модели учитывают случайные изменения суточного максимума в реальных условиях работы ЭЭС.

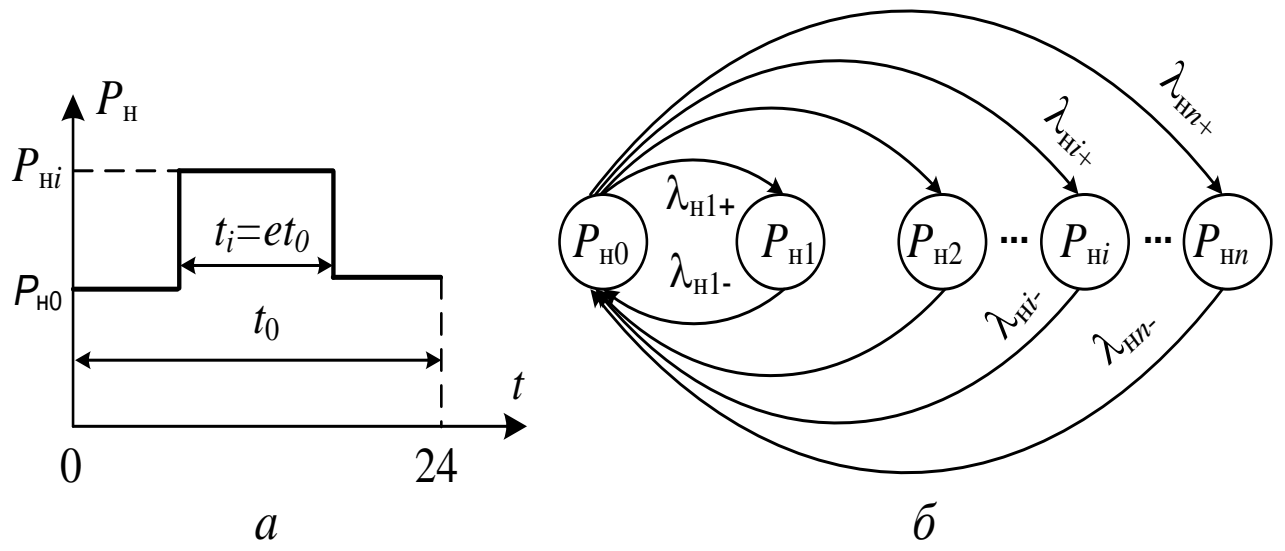

Рис. 2. Двухуровневый суточный график нагрузки (a) и его модель (б)

Fig. 2. Two-level daily load chart (a) and its model (b)

Среднюю продолжительность максимальной нагрузки $t_{i}$ определяют экспозиционным параметром $e=t_{i} / t_{0}$, где $t_{0}-$ период цикла нагрузки (в данном случае - сутки). Значение $e$ выбирают по конфигурации реального графика, что позволяет установить интенсивности переходов из состояния минимальной нагрузки в разные состояния максимальной нагрузки и обратно в марковской модели процесса изменения нагрузки

$$
\begin{gathered}
\lambda_{\mathrm{H} i+}=\alpha_{i} 1 /\left(t_{0}-t_{i}\right)=\alpha_{i} 1 /\left((1-e) t_{0}\right) ; \\
\lambda_{\mathrm{H} i-}=1 / t_{i}=1 /\left(e t_{0}\right) ; \sum_{i=1}^{n} \alpha_{i}=1,
\end{gathered}
$$

где $\alpha_{i}$ - относительные частоты возникновения соответствующих максимальних нагрузок $P_{\text {нi }}$.

Для вычисления вероятности $p_{н}$ непокрытия нагрузки системы и ожидаемого в году количества суток $n_{t}$ непокрытой нагрузки используют марковскую модель системы генерирования мощности и характеристики нагрузки как случайной величины. Для каждого $s$-го состояния системы генерирования, в котором рабочая мощность агрегатов равна $P_{c s}$, можно по графику суммарных значений (рис. 3) определить часть времени $t_{\text {нs }}^{*}$, в течении которого нагрузка превышает рабочую мощность.

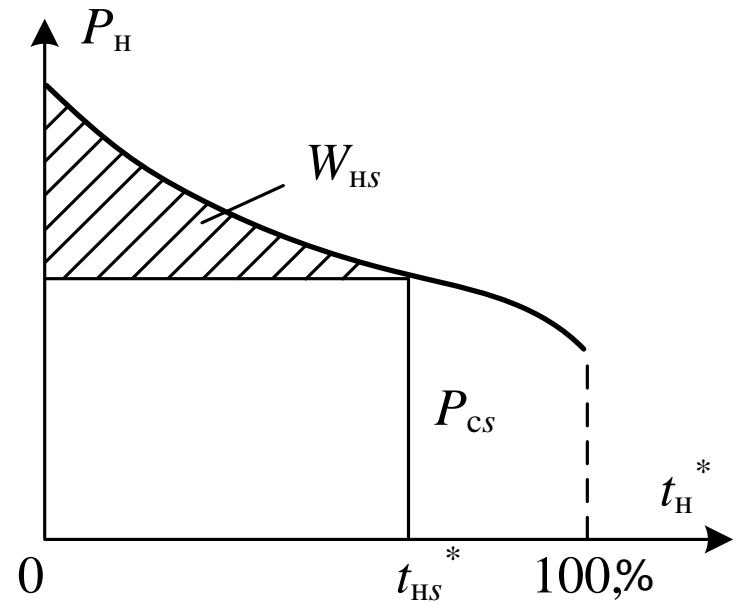

Рис. 3. Использование графика по продолжительности нагрузки

Fig. 3. Using a graph of the duration of the load

Если операцию выполнить для всех состояний системы генерирования, то вероятность $p_{\text {н }}$ непокрытия нагрузки можно вычислить следующим способом

$$
p_{\mathrm{H}}=\sum_{s \in S} p\left(P_{\mathrm{c}}=P_{\mathrm{c} s}\right) p\left(P_{\mathrm{H}}>P_{\mathrm{c} s}\right)=\sum_{s \in S} p_{s} t_{\mathrm{H} S}^{*} / 100,
$$

где $P_{\mathrm{s}}-$ вероятность $s$-го объединенного состояния системы генерирования; $S$ - общая совокупность объединенных состояний. 
Если для расчетов использовать гистограмму относительных частот нагрузки, то

$$
p_{\mathrm{H}}=\sum_{i \in I} p\left(P_{\mathrm{H} i}\right) p\left(P_{\mathrm{c}}<P_{\mathrm{H} i}\right)=\sum_{i \in I} p_{\mathrm{H} i}^{*} / 100 \sum_{P_{\mathrm{cs}}<P_{\mathrm{H} i}} p_{s},
$$

где $I$ - суммарное количество интервалов гистограммы; $p\left(P_{\mathrm{c}}<P_{\text {ні }}\right)-$ вероятность того, рабочая мощность системы генерирования будет меньше средней нагрузки $i$-го интервала гистограммы, которая равна сумме вероятностей состояний модели системы генерирования, для которых справедливо неравенство $P_{\mathrm{cs}}<P_{\mathrm{H} i}$.

Вычисление усредненного дефицита мощности $P_{\partial}$ и недоотпущенной потребителям электроэнергии. Если используется модель нагрузки в виде годового графика по продолжительности, то для $s$-го объединенного состояния системы генерирования можно рассчитать недоотпущенную электроэнергию $W_{\text {нs }}$ (рис. 3) и средний дефицит мощности $P_{\partial s}$ в течение одного цикла изменения нагрузки

$$
P_{\partial s}=W_{\mathrm{H} s} / T_{\mathrm{u}}=1 / 100 \int_{0}^{t_{\mathrm{Hs}}^{*}}\left(P_{\mathrm{H}}(t)-P_{\mathrm{cs}}\right) d t_{\mathrm{H}}^{*},
$$

где $T_{\text {ц }}$ - продолжительность цикла изменения нагрузки в часах; $P_{\mathrm{H}}(t)-$ изменение нагрузки по графику; $P_{\text {cs }}$ - мощность системы генерирования в $s$-м объединенном состоянии.

Рассчитав $P_{\partial s}$ для каждого $s$-го состояния, можно определить среднее значение дефицита и недоотпущенную электроэнергию за весь цикл изменения нагрузки с учетом всех $S$ состояний системы генерирования мощности

$$
P_{\partial}=\sum_{s \in S} P_{\partial s} p_{s} ; \quad W_{\mathrm{H}}=P_{\partial} T_{\Perp} .
$$

Не сложен и алгоритм вычисления среднего дефицита и недооппущенной электроэнергии в случае использования гистограммы относительных частот. Здесь для $i$-го интервала гистограммы

$$
P_{\partial i}=\sum_{s \in S}\left(P_{\mathrm{нi}}-P_{\mathrm{cs}}\right) p_{s},
$$

а за весь цикл изменения нагрузки

$$
P_{\partial}=\sum_{i \in I} P_{\partial i} p\left(P_{\mathrm{н} i}\right)=\sum_{i \in I} P_{\partial i} p_{\mathrm{H} i}^{*} / 100 ; \quad W_{\mathrm{H}}=P_{\partial} T_{\mathrm{u}} .
$$

Параметр потока отказов системы определяется по формуле

$$
Z_{\mathrm{c}}=\sum_{k \in V} p_{k} \cdot \sum_{l \in W} \lambda_{k l},
$$

где $\lambda_{k l}$ - интенсивность перехода из состояния $k$ в области $V$ дефицита мощности в некоторое $l$-e состояние модели, расположенное за границами области дефицита, то есть в области $W$ нормальной работы системы.

Установим вначале среднее за расчетный период значение резерва (избытка) мощности $P_{\mathrm{p} j}$ некоторой $j$-й ЭЭС. Если нагрузка этой $j$-й ЭЭС задана графиком по продолжительности, то величину $P_{\mathrm{p} j}$ определяют по соотношениям

$$
P_{\mathrm{p} j s}=(1 / 100) \int_{t_{\mathrm{m} j s}^{*}}^{100}\left(P_{\mathrm{c} j s}-P_{\mathrm{rj}}(t)\right) d t_{\mathrm{\mu j} j}^{*} ; P_{\mathrm{p} j}=\sum_{s_{j} \in S_{j}} P_{\mathrm{p} j s} p_{s} .
$$

Если нагрузка $j$-й ЭЭС задана гистограммой относительных частот, то для определения величины резерва можно воспользоваться соотношениями

$$
P_{\mathrm{p} j i}=\sum_{s_{j} \in S_{j}}\left(P_{\mathrm{c} j s}-P_{\mathrm{r} j i}\right) p_{j s} ; \quad P_{\mathrm{p} j}=\sum_{i_{j} \in I_{j}} P_{\mathrm{p} j i} p_{\mathrm{r} j i}^{*} / 100 .
$$

В случае если ЭЭС, для которой определяют показатели надежности, непосредственно связана c $J$ другими ЭЭС, то при неограниченной пропускной способности межсистемных связей величина дополнительной мощности, которая может поступать от всех $J$ электроэнергетических систем будет равна

$$
P_{\mathrm{p}}=\sum_{j \in J} P_{\mathrm{p} j} .
$$

Если пропускная способность межсистемных связей ограничена, то

$$
P_{\mathrm{p}}=\sum_{j \in J} P_{\mathrm{p} j}^{\prime}=\sum_{j \in J}\left(P_{n \mathrm{pj}}-P_{б j}\right),
$$

где $P_{n \mathrm{pj}}, P_{\text {бj }}$ - пропускная способность связи и величина балансового перетока между $j$-й и исследуемой ЭЭС.

Вероятность $p_{\text {н }}$ непокрытия нагрузки исследуемой ЭЭС можно рассчитать по формуле $p_{\mathrm{H}}=\sum_{s \in S} p\left(P_{\mathrm{c}}=P_{\mathrm{c} s}\right) p\left(P_{\mathrm{H}}>P_{\mathrm{c} s}+P_{\mathrm{p}}\right)=\sum_{s \in S} p_{s} t_{\mathrm{нsp}}^{*} / 100$.

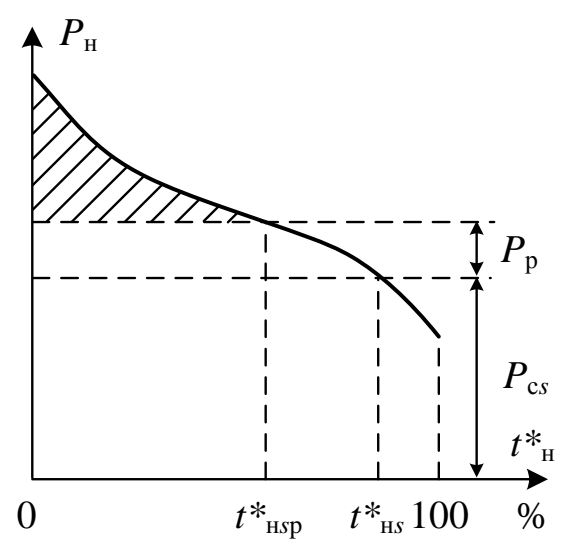

Рис. 4. Определение относительного времени непокрытия нагрузки

Fig. 4. Determination of the relative time of non-cover load 
Показанное на рис. 4 снижение относительного времени $t_{\text {нsp }}^{*}$ свидетельствует об уменьшении вероятности $p_{\text {н }}$ непокрытия нагрузки в ЭЭС, которая работает в составе энергообъединения, по сравнению изолированно работающей ЭЭС.

Вероятность $p_{\text {н }}$ непокрытия нагрузки ЭЭС в случае, если нагрузка задана гистограммой относительных частот, определяется по соотношению

$$
\begin{aligned}
p_{\mathrm{H}} & =\sum_{i \in I} p\left(P_{\mathrm{H} i}\right) p\left(P_{\mathrm{cs}}+P_{\mathrm{p}}<P_{\mathrm{н} i}\right)= \\
& =\sum_{i \in I}\left(p_{\mathrm{H} i}^{*} / 100\right) \sum_{P_{\mathrm{cs}}<P_{\mathrm{H} i}-P_{\mathrm{p}}} p_{s} .
\end{aligned}
$$

В данном случае величина $p_{\text {н }}$ уменьшается в сравнении с аналогичной величиной для изолированно работающей ЭЭС за счет уменьшения вероятности $p_{s}$.

Значения усредненного дефицита мощности $P_{\partial}$ и недоотпущенной потребителям электроэнергии $W_{\text {н }}$ при объединении ЭЭС определяют по соотношениям:

нагрузка задана графиком по продолжительности -

$$
\begin{aligned}
& P_{\partial s}=\int_{0}^{t_{\mathrm{Hsp}}^{*}}\left(P_{\mathrm{H}}(t)-P_{\mathrm{c} s}-P_{\mathrm{p}}\right) d t_{\mathrm{H}}^{*} ; \\
& P_{\partial}=\sum_{s \in S} P_{\partial s} p_{s} ; \quad W_{\mathrm{H}}=P_{\partial} T_{ц} ;
\end{aligned}
$$

нагрузка задана

гистограммой относительных частот -

$$
\begin{gathered}
P_{\partial i}=\sum_{s \in S}\left(P_{\mathrm{H} i}-P_{\mathrm{c} s}-P_{\mathrm{p}}\right) p_{s} ; \\
P_{\partial}=\sum_{i \in I} P_{\partial i} p\left(P_{\mathrm{H} i}\right)=\sum_{i \in I} P_{\partial i} p_{\mathrm{H} i}^{*} / 100 ; \quad W_{\mathrm{H}}=P_{\partial} T_{ц} .
\end{gathered}
$$

Упрощенная методика

определения балансовой надежности.

В условиях функционирования рынка электрической энергии определение балансовой надежности ЭЭС приобретает определенные особенности и несколько усложняется. Это обусловлено в частности изменением алгоритма определения состава и мощности определенной части источников генерации (работа рынка по модели Пула или механизм заключения прямых двусторонних договоров с балансирующим рынком), а также состава источников мощности для балансирования [3].
Упрощенная методика позволяет по значениям вероятности $p_{\text {c }}$ отказа системы оценить, например, значение вероятности $p_{\text {н }}$ непокрытия нагрузки. Вероятность состояния отказа системы $p_{\text {c }}$ - это относительное время, в течение которого система находится в состоянии отказа, то есть не может обеспечить покрытие нагрузки. Фактически $p_{\text {с }}$ и $p_{\text {н }}$ - это одинаковые величины, но поскольку для их расчета используют разные формы представления нагрузок, то и числовые значения оказываются разными.

Величину $p_{\text {н }}$ определяют с учетом того, что в случае непокрытия нагрузки на произвольном интервале времени в течение суток все эти сутки автоматически зачисляются во временные интервалы, в течение которых нагрузка считается непокрытой. Поэтому для $e=1$ расчетные значения вероятностей $p_{\text {c }}$ и $p_{\text {н }}$ совпадают и в общем случае

$$
p_{\mathrm{H}}=p_{\mathrm{c}} / e .
$$

Приближенно можно определить также усредненный за расчетный период $T_{\text {ц }}$ дефицит мощности в ЭЭС или в объединенной энергосистеме и недоотпущенную потребителям электроэнергию. Эти показатели определяют по известным для каждого объединенного состояния $\mu \nu$ значениям вероятности $p_{\mu \nu}$ и дефицита $p_{\partial \mu \nu}$. За период работы $T_{\text {ц }}$, для которого создавались модели генерирующей части и нагрузки

$$
\begin{gathered}
P_{\partial A}=\sum_{\mu \nu \in Д_{\partial A}} P_{\partial A \mu v} p_{\mu v} ; \quad P_{\partial B}=\sum_{\mu \nu \in Д_{\partial B}} P_{\partial B \mu v} p_{\mu v} ; \\
P_{\partial}=\sum_{\mu v \in Д_{\partial}} P_{\partial \mu \nu} p_{\mu v} .
\end{gathered}
$$

На рис. 5 приведены графики упрощенной модели балансовой надежности (а) и график по продолжительности, иллюстрирующие принцип применения упрощенной методики при определении балансовой надежности [4]. На рис. 5 обозначено: $K$ - суммарная установленная мощность блоков; $g$ - суммарная мощность отключенных блоков, находящихся в ремонте, либо в состоянии останова в результате действия рыночных механизмов; $L-$ экономически обоснованный (рыночный) спрос на мощность. В этом случае величина $L+g$ определяется как «расширенная» нагрузка, которая фактически соответствует искомому значению непокрытой нагрузки потребителей. 


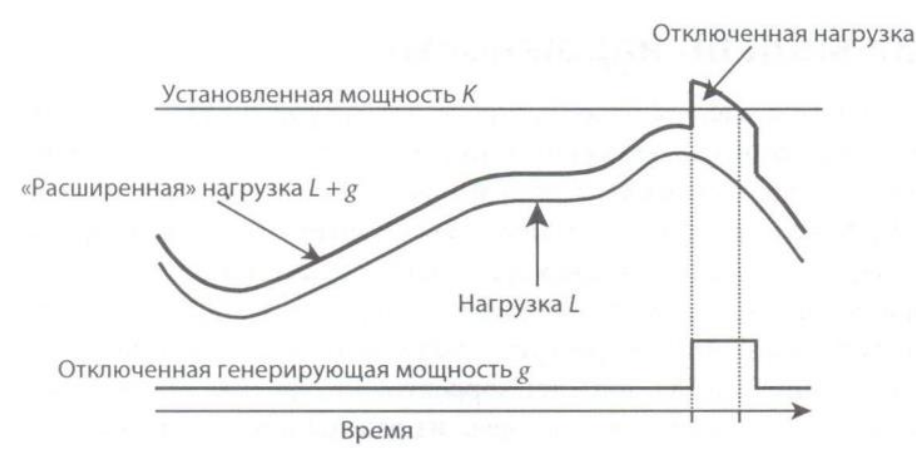

a

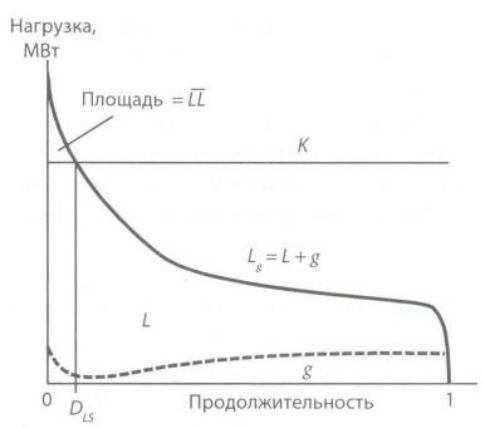

б

Рис. 5. Графики упрощенной модели балансовой надежности (a) и график по продолжительности «расширенной» загрузки (б)

Fig. 5. Graphs of a simplified model of balance reliability (a) and a graph of the duration of the «extended» load $(b)$

Выводы. Динамичный рост количества и установленной мощности возобновляемых источников энергии приводит к возрастанию вероятности нарушения балансовой надежности ЭЭС. Правила функционирования рынка электрической энергии вносят дополнительные особенности и режимные ограничения. Использование упрощенной методики анализа балансовой надежности ЭЭС позволит своевременно определить режимные нарушения и повысить надежность функционирования ЭЭС co значительной долей возобновляемых источников энергии.

1. Казанський С.В., Матеєнко Ю.П., Сердюк Б.М. Надійність електроенергетичних систем. навч. посібник. Київ. НТУУ «КПІ». 2011. 216 с. ISBN 978-966-622-453-1.

2. Журахівський А.В., Казанський С.В., Матеєнко Ю.П., Пастух О.P. Надійність електроенергетичних систем і електричних мереж: підручник. Київ. НТУУ «КПІ ім. Ігоря Сікорського». 2017. 456 с. ISBN 978-966-622-862-1.

3. Казанський С.В. Моделі організації ринків електричної енергії. Електропанорама. 2008. № 3. С. 55-57.

4. Стофт C. Экономика энергосистем. Введение в проектирование рынков электроэнергии. М. Мир. 2006. 623 с. ISBN 5-03-003439-0.

\section{REFERENCES}

1. Kazansky S.V., Mateyenko Y.P., Serdyuk B.M. Nadiinist elektroenerhetychnykh system. [Reliability of power systems]. teach. guidances. Kyiv. NTUU “KPI”. 2011. 216 p. [in Ukrainian]. ISBN 978-966-622-453-1.

2. Zhurakhivsky A.V., Kazansky S.V., Mateyenko Y.P., Pastukh O.R. Nadiinist elektroenerhetychnykh system i elektrychnykh merezh. [Reliability of power systems and power networks]. textbook. Kyiv. NTUU "Igor Sikorsky Kyiv Polytechnic Institute". 2017. 456 p. [in Ukrainian]. ISBN 978-966-622-862-1.

3. Kazansky S.V. Modeli orhanizatsii rynkiv elektrychnoi enerhii [Models of organization of markets of electric energy]. Elektropanorama. 2008. № 3. Pp. 55-57. [in Ukrainian].

4. Stoft S. Ekonomika energosistem. Vvedeniye v proyektirovaniye rynkov elektroenergii. [Power system economics. Designing markets for electricity]. M. Mir. 2006. 623 p. [in Russian]. ISBN 5-03-003439-0.

\section{ЗАБЕЗПЕЧЕННЯ БАЛАНСОВОЇ НАДІЙНОСТІ ЕЛЕКТРОЕНЕРГЕТИЧНИХ СИСТЕМ 3 ВІДНОВЛЮ- ВАНИМИ ДЖЕРЕЛАМИ ЕНЕРГІЇ}

С.В. Казанський, канд. техн. наук, доцент, Ю.П.

Матеєнко, канд. техн. наук, доцент

Національний технічний університет України «Київський політехнічний інститут ім. Ігоря Сікорського», 03056 м. Київ, пр-т Перемоги, 37

Дано визначення оперативної та балансової надійності як двох складових надійності функиіонування електроенергетичних систем (EEC). Зазначено, що балансова надійність - ие спроможність ЕEC постійно підтримувати баланс активної потужності та забезпечувати постачання потужності спожсивачам з урахуванням ймовірних відключень елементів ЕЕС. Показано необхідність забезпечення балансової надійності ЕЕС та енергооб'єднань як єдиного об'єкта керування. При иъьоу балансова надійність є об'єктивною характеристикою стану генерувальної частини ЕEC $і$ критеріси ймовірності обмежсення постачання потужності споживачам. Показано взаємозв'язок між збільшенням кількості відновлюваних джерел енергї̈ та зростанням ймовірності зниження балансової надійності $E E C$ з обмежкенням потужності генерувальних агрегатів або пропускної спроможності електричних мереж. Наведено показники визначення балансової надійності ЕЕС. Показано послідовність визначення ймовірності незабезпечення навантаження системи, засновану на використанні марковської моделі генерування потужсності. Запропоновано методику чисельного оцінювання показників балансової надійності ЕЕС з відновлюваними джсерелами енергії. Розглянуто моделі представлення навантажсення характеристиками випадкової величини або випадкового проиесу. Виконано порівняльний аналіз моделей при визначенні балансової надійності. Показано, що в умовах функціонування ринку електричної енергї зростає ймовірність порушення балансової надійності ЕЕС. Запропоновано спрощену методику оцінювання балансової надійності ЕEС зі значною кількістю відновлюваних джерел енергії, яка дозволяє за значеннями ймовірності відмови системи оцінити ймовірність непокриття навантаження на довільному інтервалі часу, а також дефіият потужності EEC. Наведено графічну інтерпретаиію спрощеної моделі забезпечення балансової надійності ЕЕС. Бібл. 4, puc. 5 .

Ключові слова: електроенергетична система, балансова надійність, відновлювані джерела енергї, ринок електричної енергії. 